\title{
Hidden-charm meson-baryon molecules with a short-range attraction from five quark states
}

\section{Yasuhiro Yamaguchi*}

RIKEN Nishina Center

E-mail: yasuhiro.yamaguchi@riken.jp

\begin{abstract}
We investigate the exotic baryons as $\bar{D}^{(*)} \Lambda_{\mathrm{c}}$ and $\bar{D}^{(*)} \Sigma_{\mathrm{c}}^{(*)}$ molecules coupled to the compact fivequark $(5 q)$ states. The coupling to the $5 q$ state is introduced by the contact term, where the relative strength for the meson-baryon channels is determined by the spin structure of the $5 q$ states. We also introduce the one pion exchange potential (OPEP). Resonant and/or bound states are found close to the hidden-charm meson-baryon thresholds. The OPEP is not strong enough to produce a state. The states are obtained, when the $5 q$ potential with the finite coupling strength is introduced. The spectrum structure is given by the spin configuration of the $5 q$ states.
\end{abstract}

XVII International Conference on Hadron Spectroscopy and Structure - Hadron2017

25-29 September, 2017

University of Salamanca, Salamanca, Spain

\footnotetext{
${ }^{*}$ Speaker.
} 


\section{Introduction}

The study of the exotic hadrons has been one of the interesting topics in nuclear and hadron physics. In 2015, the Large Hadron Collider beauty experiment ( $\mathrm{LHCb}$ ) collaboration reported two exotic baryons $P_{c}^{+}(4380)$ and $P_{c}^{+}(4450)$ in $\Lambda_{b}^{0} \rightarrow J / \psi K^{-} p$ decay $[1,2,3]$, where the masses and widths are $(M, \Gamma)=(4380 \pm 8 \pm 28,205 \pm 18 \pm 86) \mathrm{MeV}$ and $(4449.8 \pm 1.7 \pm 2.5,39 \pm 5 \pm 19) \mathrm{MeV}$, respectively. The decay process indicated that they are the hidden-charm pentaquarks with the minimal quark content $c \bar{c} u u d$. The spin-parity $J^{P}$ of these states has not yet been determined, but $\left(J_{P_{c}^{+}(4380)}^{P}, J_{P_{c}^{+}(4450)}^{P}\right)=\left(3 / 2^{-}, 5 / 2^{+}\right)$gives the best fit solution, and $\left(3 / 2^{+}, 5 / 2^{-}\right)$and $\left(5 / 2^{-}, 3 / 2^{+}\right)$ are also acceptable. There have been various discussions for the structure of the hidden-charm pentaquarks. The compact structure $c \bar{c} q q q$ has been studied by the quark model calculations. On the other hand, the reported masses of $P_{\mathrm{c}}$ 's being close to the $\bar{D} \Sigma_{\mathrm{c}}^{*}$ and $\bar{D}^{*} \Sigma_{\mathrm{c}}$ could indicate that the $P_{\mathrm{c}}$ 's are the hadronic molecules of $\bar{D} \Sigma_{\mathrm{c}}^{*}$ and $\bar{D}^{*} \Sigma_{\mathrm{c}}$. There have also been the non-resonant explanations of the experimental data given by the kinematical effect and cusp. These discussions are summarized in reviews in Refs. $[4,5,6]$ and references therein.

In this study, we investigate the pentaquark states as the hidden-charm molecules, $\bar{D}^{(*)} \Lambda_{\mathrm{c}}$ and $\bar{D}^{(*)} \Sigma_{\mathrm{c}}^{(*)} 1$ coupled to the compact five-quark (5q) states. The inclusion of the $5 q$ state is inspired by the recent work discussed by the quark cluster model in Ref. [7]. Coupling to the $5 q$ states is treated as the short-range interaction between the meson and the baryon, where this interaction is described by the contact term with the Gaussian form factor. As for the long-range interaction, the one pion exchange potential (OPEP) is introduced, which is enhanced by the heavy quark symmetry in the heavy flavor sector. The bound and resonant states of the meson-baryon systems are studied by solving the coupled channel Schödinger equation.

\section{Interactions}

It has been well-known that the OPEP is a driving force to bind atomic nuclei. In the heavy quark sector, the role of the OPEP would be enhanced by the heavy quark spin symmetry, where the mass degeneracy of the heavy hadron with different spin is obtained $[8,9]$. In fact, the mass difference of $\bar{D}$ and $\bar{D}^{*}$ mesons is small, $m_{\bar{D}^{*}}-m_{\bar{D}} \sim 140 \mathrm{MeV}$. The approximate mass degeneracy enhances the attraction due to the mixing of the $\bar{D}$ meson and the $\bar{D}^{*}$ meson caused by the OPEP.

The OPEP is obtained by the effective Lagrangians for heavy mesons (baryons) and the Nambu-Goldstone boson, satisfying the heavy quark and chiral symmetries $[9,10]$. From those Lagrangians, one obtains the OPEP for channel $(i, j)$ as

$$
V_{i j}^{\pi}=G_{i j}\left[\overrightarrow{\mathscr{S}}_{i} \cdot \overrightarrow{\mathscr{S}}_{j} C(r)+\mathscr{T}_{\mathscr{S}_{i} \mathscr{S}_{j}}(\hat{r}) T(r)\right],
$$

with the coupling constant $G_{i j}$, the spin operator $\overrightarrow{\mathscr{S}}_{i}$, the tensor operator $\mathscr{T}_{\mathscr{S}_{i} \mathscr{S}_{j}}(\hat{r})$, the central function $C(r)$ and the tensor function $T(r)$ defined in Ref. [11]. The channels $i$ are given by $\bar{D} \Lambda_{\mathrm{c}}\left({ }^{2} S\right), \bar{D}^{*} \Lambda_{\mathrm{c}}\left({ }^{2} S,{ }^{4} D\right), \bar{D} \Sigma_{\mathrm{c}}\left({ }^{2} S\right), \bar{D} \Sigma_{\mathrm{c}}^{*}\left({ }^{4} D\right), \bar{D}^{*} \Sigma_{\mathrm{c}}\left({ }^{2} S,{ }^{4} D\right), \bar{D}^{*} \Sigma_{\mathrm{c}}^{*}\left({ }^{2} S,{ }^{4} D,{ }^{6} D\right)$ for $J^{P}=1 / 2^{-}$.

For the $5 q$ potential, we employ the contact interaction with the Gaussian form factor,

$$
V^{5 q}=-f S_{i} S_{j} e^{-\alpha r^{2}} .
$$

\footnotetext{
${ }^{1}$ Here, $\bar{D}^{(*)}\left(\Sigma_{\mathrm{c}}^{(*)}\right)$ stands for $\bar{D}$ and $\bar{D}^{*}\left(\Sigma_{\mathrm{c}}\right.$ and $\left.\Sigma_{\mathrm{c}}^{*}\right)$.
} 
Table 1: Spectroscopic factor of the $5 q$ potential for $J^{P}=1 / 2^{-} . S_{c \bar{c}}\left(S_{3 q}\right)$ is the total spin of $c \bar{c}$ (three light quarks $q q q$ ).

\begin{tabular}{ccc|cccccc}
\hline \hline & $S_{c \bar{c}}$ & $S_{3 q}$ & $\bar{D} \Lambda_{\mathrm{c}}$ & $\bar{D}^{*} \Lambda_{\mathrm{c}}$ & $\bar{D} \Sigma_{\mathrm{c}}$ & $\bar{D} \Sigma_{\mathrm{c}}^{*}$ & $\bar{D}^{*} \Sigma_{\mathrm{c}}$ & $\bar{D}^{*} \Sigma_{\mathrm{c}}^{*}$ \\
\hline (i) & 0 & $1 / 2$ & 0.4 & 0.6 & -0.4 & - & 0.2 & -0.6 \\
(ii) & 1 & $1 / 2$ & 0.6 & -0.4 & 0.2 & - & -0.6 & -0.3 \\
(iii) & 1 & $3 / 2$ & 0.0 & 0.0 & -0.8 & - & -0.5 & 0.3 \\
\hline \hline
\end{tabular}

The negative sign of $V^{5 q}$ is assumed because of the massive compact $5 q$ state giving an attraction [11]. The strength $f$ and Gaussian range $\alpha$ are treated as a free parameter. The range $\alpha$ is fixed as $\alpha=1 \mathrm{fm}^{-2}$ in this study, while the energies for various strength $f$ will be shown in Sec. 3 . The spectroscopic factor (S-factor) $S_{i}$ is determined by the overlap of wavefunctions of the $5 q$ state and the meson-baryon molecule as $S_{i}=\left\langle\left(\bar{D} Y_{\mathrm{c}}\right)_{i} \mid 5 q\right\rangle$. When the spin structure of the $5 q$ state is described by the $c \bar{c}$ and $q q q$ spins $\left(S_{c \bar{c}}\right.$ and $\left.S_{3 q}\right)$, the $\mathrm{S}$-factor is obtained as shown in Table 1. For $J^{P}=1 / 2^{-}$with zero orbital momentum ( $S$-wave), there are three possible spin configurations, (i) $\left(S_{c \bar{c}}, S_{3 q}\right)=(0,1 / 2)$, (ii) $(1,1 / 2)$ and (iii) $(1,3 / 2)$. In the Table 1 , one can find components having a large S-factor, e.g. \pm 0.6 in $\bar{D}^{*} \Lambda_{\mathrm{c}}$ and $\bar{D}^{*} \Sigma_{\mathrm{c}}^{*}$ in (i). We expect that these channels play an important role to produce an attraction.

\section{Numerical results}

Solving the coupled channel Schödinger equation for $\bar{D}^{(*)} \Lambda_{\mathrm{c}}$ and $\bar{D}^{(*)} \Sigma_{\mathrm{c}}^{(*)}$ with the OPEP and the $5 q$ potential, we obtain the bound and resonant states as shown in Fig. 1. We show the energies with various coupling constant $f$. We obtain no state in the small $f$ region, while the states appear as $f$ is increased. Therefore, the OPEP is not enough to produce any state in the hidden-charm mesonbaryon systems for $J^{P}=1 / 2^{-}$. The states are obtained close to the meson-baryon thresholds as $f$ is increased. Then, the thresholds correspond to the meson-baryon components with large S-factor as shown in Table 1. The spectrum structure of the states is given by the S-factor is determined by the spin structure of the compact $5 q$ states.

\section{Summary}

We have studied the hidden-charm meson-baryon system as $\bar{D}^{(*)} \Lambda_{\mathrm{c}}$ and $\bar{D}^{(*)} \Sigma_{\mathrm{c}}^{(*)}$ coupled to the compact $5 q$ states. The coupling to the $5 q$ state is introduced by the Gaussian potential as a short range force, where the relative strength for the meson-baryon channels is given by the spectroscopic factor determined by the spin wavefunction of the $5 q$ states. The Gaussian potential plays an attractive force because of the massive $5 q$ state, while the coupling strength and Gaussian range are treated as a free parameter. As for a long range force, the one pion exchange potential is introduced. We has obtained binding and resonance energies with various coupling $f$. No state is obtained when we use only the OPEP. However, the states appear, when the $5 q$ potential with the finite coupling strength is introduced. The energy spectra are obtained close to the meson-baryon thresholds corresponding to the channels with the large S-factor. 

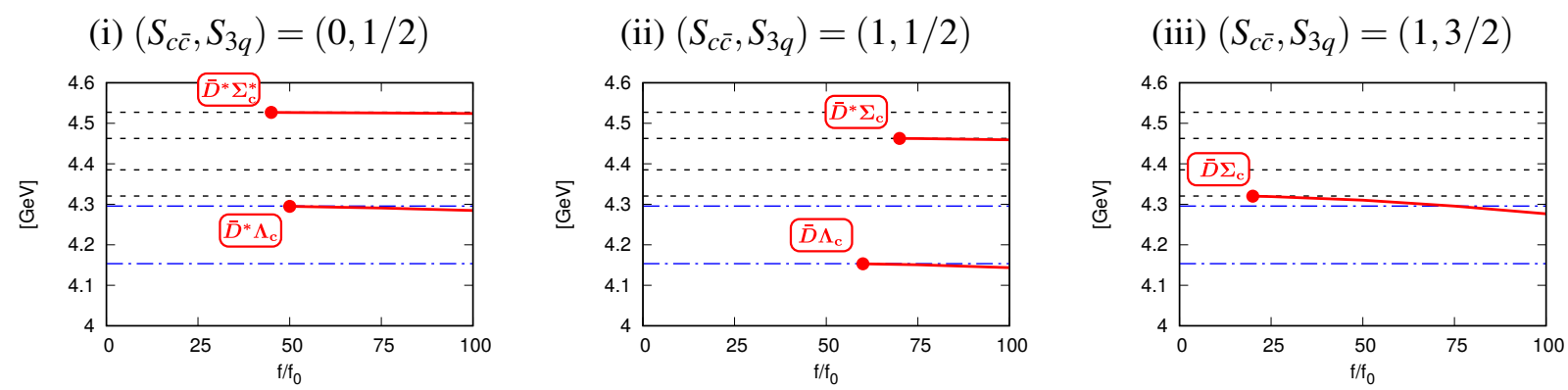

Figure 1: Obtained binding and resonance energies (solid lines) with various coupling constants $f$ for $J^{P}=1 / 2^{-}$As for interactions, the OPEP and one of the three $5 q$ potentials derived from the configuration (i) $\left(S_{c \bar{c}}, S_{3 q}\right)=(0,1 / 2)$, (ii) $(1,1 / 2)$ or (iii) $(1,3 / 2)$ are used. The horizontal axis shows the ratio $f / f_{0}$, where $f_{0}$ is the reference value, $f_{0} \sim 6 \mathrm{MeV}$. Filled circle is the starting point where the states appear. Dashed lines are the $\bar{D} \Sigma_{\mathrm{c}}, \bar{D} \Sigma_{\mathrm{c}}^{*}, \bar{D}^{*} \Sigma_{\mathrm{c}}$ and $\bar{D}^{*} \Sigma_{\mathrm{c}}^{*}$ thresholds. Dot-dashed lines are the $\bar{D} \Lambda_{\mathrm{c}}$ and $\bar{D}^{*} \Lambda_{\mathrm{c}}$ thresholds.

\section{Acknowledgments}

This work is supported by the Special Postdoctoral Researcher (SPDR) Program of RIKEN.

\section{References}

[1] R. Aaij et al. [LHCb Collaboration], Phys. Rev. Lett. 115, 072001 (2015).

[2] R. Aaij et al. [LHCb Collaboration], Phys. Rev. Lett. 117, 082002 (2016).

[3] R. Aaij et al. [LHCb Collaboration], Phys. Rev. Lett. 117, 082003 (2016); Phys. Rev. Lett. 117, 109902 (2016); Phys. Rev. Lett. 118, 119901 (2017).

[4] H. X. Chen, W. Chen, X. Liu, and S. L. Zhu, Phys. Rept. 639, 1 (2016).

[5] A. Esposito, A. Pilloni and A. D. Polosa, Phys. Rept. 668, 1 (2016).

[6] A. Ali, J. S. Lange and S. Stone, Prog. Part. Nucl. Phys. 97, 123 (2017).

[7] S. Takeuchi and M. Takizawa, Phys. Lett. B 764, 254 (2017).

[8] N. Isgur and M. B. Wise, Phys. Lett. B 232, 113 (1989).

[9] A. V. Manohar and M. B. Wise, Heavy Quark Physics, (Cambridge University Press, Cambridge, 2000), Cambridge Monographs on Particle Physics, Nuclear Physics and Cosmology, p. 191.

[10] T. M. Yan, H. Y. Cheng, C. Y. Cheung, G. L. Lin, Y. C. Lin, and H. L. Yu, Phys. Rev. D 46, 1148 (1992) Erratum: [Phys. Rev. D 55, 5851 (1997)].

[11] Y. Yamaguchi, A. Giachino, A. Hosaka, E. Santopinto, S. Takeuchi and M. Takizawa, arXiv:1709.00819 [hep-ph]. 